\section{What are the half-lives of ranibizumab and aflibercept (VEGF Trap-eye) in human eyes? Calculations with a mathematical model}

\author{
Michael W. Stewart \\ Mayo Clinic School of Medicine, \\ Jacksonville, FL, USA
}

\begin{abstract}
The aim of the article is to estimate the intravitreal half-lives of ranibizumab and aflibercept (VEGF Trap-eye; VTE) in human eyes. Using a published mathematical model for rabbits, the intravitreal half-lives of ranibizumab and bevacizumab were calculated and compared to empirical data. The slope coefficient within the model was changed to set the bevacizumab output equal to experimental values to meet 3 goals: firstly, to validate the model in rabbit eyes; secondly, to test the mutability of the model to monkey eyes; thirdly, to calculate the half-lives of ranibizumab and the VTE in human eyes. The half-life calculations for ranibizumab deviate from published rabbit and monkey values by only $8.3 \%$ and $4.2 \%$. Using the experimentally determined half-life of bevacizumab in human eyes ( 8.25 days) to set the equation, the half-lives of ranibizumab and the VTE are calculated to be 4.75 days and 7.13 days in human eyes. The intraocular half-lives of ranibizumab and the VTE are estimated using existing published animal and human data and a mathematical model. The validity of these half-lives and binding activities, however, awaits clinical correlation.
\end{abstract}

\section{Introduction}

Antibody based anti-VEGF drugs have become standard of care for the treatment of exudative age-related macular degeneration and are frequently administered for diabetic retinopathy and retinal vein occlusions. The VIEW studies showed that ranibizumab and the aflibercept (VEGF Trap-eye; VTE) had comparable peak clinical effects (Heier J, Presentation, Angiogenesis, Miami, FL, February 11, 2011; Smith Urfurth U, Presentation, Angiogenesis, Miami, FL, February 11, 2011) suggesting that the maximum clinical response achievable with anti-VEGF monotherapy in a study population may have been reached by the currently available drugs. New anti-VEGF agents may need to be differentiat- ed more by their duration of action than by their peak clinical effect.The duration of clinical action of anti-VEGF drugs is determined by a combination of binding strength and intraocular half-life. ${ }^{1,2}$ The intravitreal half-life of most drugs is first determined in animal models, usually rabbit and/or monkey, and then in humans during phase I-III trials or postapproval. Pharmacokinetic studies in human eyes usually consist of intravitreal drug injections followed several days to weeks later by sampling of the vitreous or aqueous during a surgical procedure. For most drugs, pharmacokinetic data in at least 1 animal model has been determined and some, such as ranibizumab, receive regulatory approval without human pharmacokinetic studies. ${ }^{3,4}$

The goal of this study is to estimate the as yet undetermined intravitreal half-lives of ranibizumab and the VTE in humans by using experimental animal and human data combined with a previously published mathematical model. ${ }^{5}$

\section{Materials and Methods}

A Medline search for studies reporting pharmacokinetic data on ranibizumab, bevacizumab, the VTE and similar macromolecules in both animals and humans was performed. Unique animal models - rabbit and monkey that tested both ranibizumab and bevacizumab or structurally similar molecules were identified..$^{6-8}$ The intravitreal half-life of bevacizumab in humans was calculated by averaging values from published studies. ${ }^{9-10}$ A previously published mathematical model that calculated intravitreal drug half-lives in rabbits was employed. ${ }^{5}$ The model predicts the half-life of a drug according to the following equation:

$$
\begin{gathered}
\log \mathrm{t}_{1 / 2}=-0.32+0.432^{*}(\log \mathrm{MW})-0.157^{*} \\
(\log \mathrm{P})+0.003^{*}(\text { dose / solubility) at } \mathrm{pH} 7.4
\end{gathered}
$$

where:

$t_{1 / 2}$ is the half-life of the compound.

MW is the molecular weight of the compound. Log $\mathrm{P}$ is the logarithm of $\mathrm{P}$, the partition coefficient or the ratio of the concentrations of an un-ionized compound in two immiscible phases at equilibrium. As such, $\log P$ is the lipophilicity of the compound and $\mathrm{P}=-0.51$ for macromolecules.

The ratio of dose/solubility is assumed to be 1 for this data set.

To test the validity of the model, the halflives of ranibizumab and bevacizumab were calculated. The slope coefficient (0.432) was changed slightly to set the half-life of bevacizumab equal to the published value, the expected half-life of ranibizumab was re-calculated, and the deviation, as a percentage from
Correspondence: Michael W. Stewart, 4500 San Pablo Rd., Jacksonville, FL 32224, USA. Tel. +1.904.953.2232 - Fax: +1.904.953.7040. E-mail: stewart.michael@mayo.edu

Key words: age-related macular degeneration, ranibizumab, bevacizumab, VEGF Trap, aflibercept, half-lives, pharmacokinetics.

Received for publication: 11 June 2011.

Accepted for publication: 31 July 2011.

This work is licensed under a Creative Commons Attribution NonCommercial 3.0 License (CC BYNC 3.0).

(C) Copyright M.W. Stewart, 2011

Licensee PAGEPress, Italy

Eye Reports 2011; 1:e5

doi:10.4081/eye.2011.e5

the published value, was calculated. The mutability of the model to monkey eyes was then tested. The slope coefficient was changed to set the bevacizumab half-life equal to the published value, the expected ranibizumab halflife was calculated, and the deviation, as a percentage from the published value, was calculated. Finally the model was used to calculate the drug half-lives in human eyes. The slope coefficient was changed to set the bevacizumab half-life equal to the average of the published values ( 8.25 days) and the half-lives of ranibizumab (MW-48 kD) and the VTE (MW$110 \mathrm{kD}$ ) were calculated. To determine the possible relationship between the size of the eye and the mutability of the equation, the slope coefficients were graphed against the intravitreal volumes and subjected to a regression analysis.

\section{Results}

Based upon a review of the literature (Table 1), ,4,6-14 the following animal models were selected against which to test the validity and mutability of the modified half-life equation: i) For rabbits, Bakri et al. determined ranibizumab and bevacizumab half-lives of 2.88 days and 4.32 days respectively; 7,8 ii) For monkeys, Mordenti et al. determined Fab and HER2 (macromolecules structurally similar to ranibizumab and bevacizumab, respectively) half-lives of 3.2 days and 5.6 days respectively. ${ }^{6}$

Using the published half-life equation, the initially calculated half-lives of ranibizumab and bevacizumab in rabbit eyes were 2.54 Days and 4.17 Days. The slope coefficient was increased from 0.43200 to 0.43564 to set the output for bevacizumab to equal 4.32 days. The re-calculated half-life of ranibizumab was 2.64 days, only $8.3 \%$ shorter than the experimental 
value (2.88 days). To establish the mutability of the half-life equation to monkey eyes, the slope coefficient was increased to 0.45720 to set the bevacizumab output to 5.6 days. The calculated half-life of ranibizumab was 3.34 days, only $4.2 \%$ above the experimental value (3.2 days). To calculate the half-lives of ranibizumab and the VTE in human eyes the slope coefficient was changed a third time. Increasing the coefficient to 0.49000 calculates the bevacizumab half-life to be 8.25 days, the average value reported in the literature. By inputting the molecular weights of ranibizumab and the VTE, their half-lives are calculated to be 4.75 days and 7.13 days. To determine a possible relationship between eye size and mutability of the equation between species, the 3 slope coefficients used in the previous calculations were graphed against eye volumes (Figure 1).

A linear relationship with a high correlation coefficient $\left(\mathrm{r}^{2}=0.9956\right)$ was determined.

\section{Discussion}

Accurate intraocular drug half-lives allow physicians to create efficacy models to predict the results of untested clinical situations and to more accurately predict drug washout periods when patients change medications or enter controlled clinical trials. A reliable mathematical model would enable physicians to design better clinical studies and provide improved patient care. Experimental pharmacokinetic data from rabbits, monkeys and humans has been published for the 3 antibody based anti-VEGF drugs but only bevacizumab has been studied in all 3 species., ${ }^{3,46-13}$ Until human data for ranibizumab and the VTE become available, half-life calculations based upon a methodically derived mathematical model may provide the most accurate values. Published reports show that intravitreal halflife differences exist even for the same drug within a single species. These differences may be due to several factors including vitreous and aqueous sampling techniques, drug reflux at the time of injection, and drug assay technique and performance. When choosing experimental models with which to make half-life comparisons between different drugs, models that test at least 2 drugs - Bakri et al.'s rabbit model and Mordenti et al.'s monkey model would minimize artifactual differences. A mathematical model derived from experimental rabbit data ${ }^{5}$ provides the best starting point for predicting drug half-lives in other species. This model was created from half-life data of over 60 drugs, including bevacizumab. According to the model the most important determinant of drug half-life is molecular size; less important factors include lipophilicity,
Table 1. Intraocular half-lives of ranibizumab, VTE, bevacizumab and similar macromolecules are listed.

\begin{tabular}{lllc}
\hline Authors & Drug & Species & Half-life \\
Bakri $^{8}$ & Ranibizumab & Rabbit & 2.88 days \\
Gaudreault $^{11}$ & Ranibizumab & Rabbit & 2.89 days \\
\hline $\begin{array}{l}\text { Regeneron } \\
\text { Bakri }^{\text {r7 }}\end{array}$ & VTE & Rabbit & 4.79 days \\
\hline Nomoto $^{12}$ & Bevacizumab & Rabbit & 4.32 days \\
Miyake $^{13}$ & Bevacizumab & Rabbit & 5.95 days \\
Kim $^{14}$ & Bevacizumab & Rabbit & 2.8 days (aqueous) \\
Gaudreault $^{4}$ & Infliximab & Rabbit & 4.7 days \\
\hline Gaudreault $^{4}$ & Ranibizumab & Monkey & 2.63 days (0.5 mg)0 \\
Mordenti $^{6}$ & Ranibizumab & Monkey & 3.9 days (2.0 mg) \\
\hline Mordenti $^{6}$ & Fab & Monkey & 3.2 days \\
Zhu $^{10}$ & HER2 & Monkey & 5.6 days \\
Krohne $^{9}$ & Bevacizumab & Human & 6.7 days \\
\hline
\end{tabular}

drug solubility, dose, salt form factor, and eye pigmentation. The model predicts that macromolecules with similar structure (e.g. Fab antibody fragments or full length antibodies) should have similar half-lives within a given species. Experimental data with rituximab (MW - $145 \mathrm{kD}$ ) - intravitreal half-life of 4.7 days in rabbits - suggests this to be true. ${ }^{14}$ Since lipophilicity, drug solubility, and salt form factor are independent of the vitreous volume, the model was altered by changing only the slope coefficient. Bevacizumab data exists for all 3 species so its half-lives were used to guide changes of the slope coefficient within the mathematical model. These changes were made with 3 goals in mind: i) To establish validity of the model in rabbits; ii) To establish mutability of the model to monkeys; iii) To calculate half-lives of ranibizumab and VTE in humans.

The bevacizumab guided changes in the model produced ranibizumab values of 2.64 days in the rabbit and 3.34 days the monkey, differing from the experimental values by only $8.3 \%$ and $4.2 \%$. This finding suggested that the model was accurate for macromolecule halflives in rabbit eyes and mutable to monkey eyes. Given these findings, the model was used to calculate the half-lives of ranibizumab ( 4.75 days) and the VTE (7.13 days) in humans. Both published experimental results and the halflife calculations obtained with this adapted model are consistent with 2 commonly held principles of intraocular drug pharmacokinetics. Firstly, the intravitreal half-life of a given drug increases with the size of the eye. When the slope coefficient is graphed against the size of the eye a highly correlated $\left(r^{2}=0.9956\right)$ linear relationship is seen. This limited experimental data suggests that the half-life of a drug in one species may be predicted based upon experimental data in other species. More work, however, needs to be done to determine

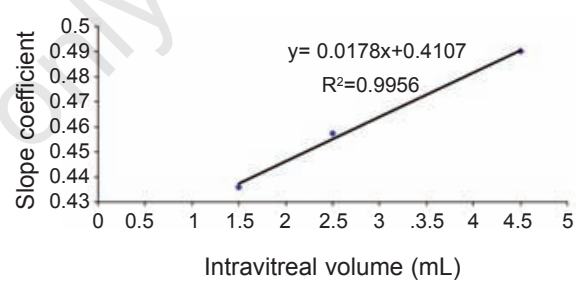

Figure 1. Intravitreal volume is graphed against the slope coefficient of the mathematical model, as determined by bevacizumab half-lives.

the validity of such a mathematical relationship. Secondly, the half-lives of drugs with similar structures increase according to the logarithm of molecular weight. This suggests that for the anti-VEGF drugs the intravitreal halflives should rank as follows: bevacizumab > VTE > ranibizumab.

The major weakness of this model concerns between species. Though changing the slope coefficient seems a logical transformation, the validity of this strategy must await confirmation with experimental data.

\section{References}

1. Stewart MW. Predicted biologic activity of intravitreal bevacizumab. Retina 2007;27: 1196-200.

2. Stewart MW, Rosenfeld P. Predicted biological activity of intravitreal VEGF Trap. Br J Ophthalmol 2008;92:667-8.

3. Gaudreault J, Webb W, Van Hoy M, et al. Pharmacokinetics and retinal distribution of AMD rhuFab V2 after intravitreal administration in rabbits. AAPS Pharm Sci 1999;Suppl 1:2142. the mutability of the rabbit-determined model 
4. Gaudreault J, Fei D, Rusit J, et al. Preclinical pharmacokinetics of ranibizumab (rhuFabV2) after a single intravitreal administration. Invest Ophthalmol Vis Sci 2005;46:726-33.

5. Durairaj C, Shah JC, Senapati S, Kompella UB. Prediction of vitreal half-life based on drug physiochemical properties: quantitative structure-pharmacokinetic relationships (QSPKR). Pharm Res 2009;26:123660.

6. Mordenti J, Cuthbertson RA, Ferrara N, et al. Comparisons of the intraocular tissue distribution, pharmacokinetics, and safety of 125I-labeled full-length and Fab antibodies in rhesus monkeys following intravitreal administration. Toxicol Pathol 1999; 27:536-44.

7. Bakri SJ, Snyder MR, Reid JM, et al.
Pharmacokinetics of intravitreal bevacizumab (Avastin). Ophthalmology 2007; 114;855-9.

8. Bakri SJ, Snyder MR, Reid JM, et al. Pharmacokinetics of intravitreal ranibizumab (Lucentis). Ophthalmology 2007;114: 2179-82.

9. Krohne TU, Eter N, Holz FG, Meyer CH. Intraocular pharmacokinetics of bevacizumab after a single intravitreal injection in humans. Am J Ophthalmol 2008; 146:508-12.

10. Zhu Q, Zeimssen F, Henke-Fahle S, et al. Vitreous levels of bevacizumab and vascular endothelial growth factor-A in patients with choroidal neovascularization. Ophthalmology 2008;115:1750-5.

11. Gaudreault J, Fei D, Beyer JC, et al. Pharmacokinetics and distribution of ranibizumab, a humanized antibody fragment directed against VEGF-A, following intravitreal administration in rabbits. Retina 2007;27:1260-6.

12. Nomoto H, Shiraga F, Kuno N, et al. Pharmacokinetics of bevacizumab after topical, subconjunctival, and intravitreal administration in rabbits. Invest Ophthalmol Vis Sci 2009;50:4807-13.

13. Miyaki T, Sawada 0, Kakinoki M, et al. Pharmacokinetics of bevacizumab and its effect on vascular endothelial growth factor after intravitreal injection of bevacizumab in macaque eyes. Invest 0phthalmol Vis Sci 2010;51:1606-8.

14. Kim H, Csaky KG, Chan CC, et al. The pharmacokinetics of rituximab following an intravitreal injection. Exp Eye Res 2006; 82:760-6. 